\title{
The changing face of HIV/AIDS care
}

\section{Laura Mesana}

\section{University of Ottawa}

With the increased usage of antiretroviral therapy (ART), HIV infection is no longer considered a death sentence and is now perceived as a chronic condition. ${ }^{1}$ HIV-positive individuals are aging with the disease and can have life expectancies that approach those of their uninfected counterparts. ${ }^{1}$ It is estimated that "by 2015, approximately $50 \%$ of people living with HIV will be older than 50 years of age." ${ }^{2}$ However, as the lifespan of HIVpositive persons increases, so does their probability of developing age-associated non-communicable diseases such as cardiovascular diseases, strokes, and cancer, all of which are considered to be major causes of death in North America. ${ }^{3}$ Furthermore, the HIV-infected population is at a heightened risk of developing these diseases due to the interaction between factors including the virus itself, ART, and senescence processes.1 This leads to the characterization of HIV/AIDS as a disease that accelerates the aging process. ${ }^{1}$ This article highlights the challenges associated with providing quality primary care to HIV-infected individuals in Canada with respect to managing chronic noncommunicable comorbidities as well as providing both comprehensive and coordinated patient care.

The main focus of primary care for the HIV-positive population should be on reducing the risk of developing age-associated comorbidities. ${ }^{4}$ The Infectious Diseases Society of America recently developed new guidelines and recommendations for providing adequate primary care to the HIV-infected population. ${ }^{4}$ They recommend that primary care physicians focus on preventive care such as screening for high cholesterol, diabetes, and osteoporosis. They add that further preventive measures targeting subsets of the HIV population should be taken. For instance, bone densitometry testing for all HIV-infected men over 50 years of age is recommended, as the osteoporosis rates are significantly higher in this specific group. Emphasizing the importance of following a balanced diet and not smoking when following an ART regimen should also be an essential component of preventive care for the HIVpositive population. Adopting such guidelines is thus crucial to palliate the age-associated non-communicable comorbidities increasingly seen in the HIV/AIDS population.

Furthermore, the new guidelines highlight the role of primary care in ensuring that patients are coping with their diagnosis by having sufficient support networks. ${ }^{4}$ This suggests that mental health should also be a priority in the primary care services offered to the HIV population. HIVpositive individuals often face discrimination and may not always have access to a reliable support system. ${ }^{5}$ These issues have been linked with depression, lower adherence to ART, and an increased susceptibility to adopt high-risk behaviors. ${ }^{6}$ For this reason, primary care providers need to adopt a holistic approach in ensuring that persons living with HIV/AIDS are not only adhering to their medications but also to a healthy lifestyle. Routinely assessing the mental health of HIV-positive patients should be a fundamental part of their primary care services, as it has the potential to improve their quality of life by motivating them to live healthier lives and optimizing their adherence to ART.

Another challenge to providing comprehensive primary care to the HIV-positive population is due to the fragmentation of the health care services currently offered to them. ${ }^{7}$ For the most part, HIV specialists assume the role of primary care providers for HIV-infected individuals because primary care physicians do not necessarily have the same level of expertise as HIV specialists and regularly need to educate themselves about treatments and the impact of HIV/AIDS on routine health care. ${ }^{8}$ This creates a gap of knowledge between primary care physicians and HIV specialists. Integrating the knowledge of the various health care professionals involved in providing primary care to HIV-positive individuals would help create a continuum of primary care providers. Bridging such gaps would increase $>$ 
the accessibility and consistency of preventive services; both essential components of cost-effective primary care.

As patients are aging and dealing with the consequences of having HIV, primary care providers should focus on preventing chronic non-communicable comorbidities. The complexity of providing primary care to the HIV-positive population stems from the fact that physicians have to palliate age-associated chronic conditions in addition to treating these patients' underlying HIV infection. The new guidelines synthesize the highest quality of evidence on the matter and aim to bridge knowledge gaps between primary care physicians and HIV specialists. However, further research should be conducted on cost-effective solutions to better structure primary care services in order to efficiently meet the needs of the aging HIV-positive population. Improving the quality of continuous primary care is essential to ensure both longevity and health-related quality of life in the Canadian HIV-infected population.

\section{References}

1. Justice AC, Braithwaite RS. Lessons learned from the first wave of aging with HIV. AIDS. 2012;26 (Suppl 1):11-8.

2. Effros RB, Fletcher CV, Gebo K, Halter JB, Hazzard WR, Horne FM, et al. Aging and infectious diseases: Workshop on HIV infection and aging: What is known and future research directions. Clin Infect Dis. 2008;47(4):542-53.

3. Hoyert DL, Xu J. Deaths: Preliminary data for 2011. National Vital Statistics Reports. 2012;61(6):1-51.

4. Aberg JA, Gallant JE, Ghanem KG, Emmanuel P, Zingman BS, Horberg MA. Primary care guidelines for the management of persons infected with HIV: 2013 update by the HIV Medicine Association of the Infectious Diseases Society of America. Clin Infect Dis. 2014;58(1):1-10.

5. Brennan DJ, Emlet CA, Eady A. HIV, sexual health, and psychosocial issues among older adults living with HIV in North America. Ageing Int. 2011;36(3):313-33.

6. Aranda-Naranjo B. Quality of life in the HIV-positive patient: Implications and consequences. The Journal of the Association of Nurses in AIDS Care: JANAC. 2004;15(5 Suppl):20S-7S.

7. Mugavero MJ, Norton WE, Saag MS. Health care system and policy factors influencing engagement in HIV medical care: Piecing together the fragments of a fractured health care delivery system. Clin Infect Dis. 2011;52 (Suppl 2):238-46.

8. Smith SM, Allwright $S$, O'Dowd T. Effectiveness of shared care across the interface between primary and specialty care in chronic disease management. Cochrane Database Syst Rev. 2007(3):CD004910.

\section{Laura Mesana}

Laura Mesana completed an undergraduate degree in Arts and Science from McGill University and is currently finishing her Masters at the University of Ottawa in the School of Interdisciplinary Health Science. Her thesis work comprises a series of systematic reviews and meta-analyses on the efficacy and safety of statins as lipid-lowering therapy for the hyperlipidemic HIV-infected population. Her primary research interests are evidence-based medicine and population health. Laura is also interested in community medicine and primary health care. 